\title{
Compact perturbative expressions for neutrino oscillations in matter
}

\author{
Peter B. Denton, ${ }^{a, b}$ Hisakazu Minakata ${ }^{c, d}$ and Stephen J. Parke ${ }^{a}$ \\ ${ }^{a}$ Theoretical Physics Department, Fermi National Accelerator Laboratory, \\ P.O. Box 500, Batavia, IL 60510, U.S.A. \\ ${ }^{b}$ Physics 8 Astronomy Department, Vanderbilt University, \\ PMB 401807, 2301 Vanderbilt Place, Nashville, TN 37235, U.S.A. \\ ${ }^{c}$ Instituto de Física, Universidade de São Paulo, \\ C.P. 66.318, 05315-970 São Paulo, Brazil \\ ${ }^{d}$ Department of Physics, Yachay Tech University, \\ San Miguel de Urcuqui 100119, Ecuador \\ E-mail: peterbd1@gmail.com, hminakata@yachaytech.edu.ec, \\ parke@fnal.gov
}

ABSTRACT: We further develop and extend a recent perturbative framework for neutrino oscillations in uniform matter density so that the resulting oscillation probabilities are accurate for the complete matter potential versus baseline divided by neutrino energy plane. This extension also gives the exact oscillation probabilities in vacuum for all values of baseline divided by neutrino energy. The expansion parameter used is related to the ratio of the solar to the atmospheric $\Delta m^{2}$ scales but with a unique choice of the atmospheric $\Delta m^{2}$ such that certain first-order effects are taken into account in the zeroth-order Hamiltonian. Using a mixing matrix formulation, this framework has the exceptional feature that the neutrino oscillation probability in matter has the same structure as in vacuum, to all orders in the expansion parameter. It also contains all orders in the matter potential and $\sin \theta_{13}$. It facilitates immediate physical interpretation of the analytic results, and makes the expressions for the neutrino oscillation probabilities extremely compact and very accurate even at zeroth order in our perturbative expansion. The first and second order results are also given which improve the precision by approximately two or more orders of magnitude per perturbative order.

Keywords: CP violation, Neutrino Physics

ARXIV EPRINT: 1604.08167 


\section{Contents}

1 Introduction 1

2 Rotations of the neutrino basis and the Hamiltonian 3

2.1 Overview 3

$2.2 U_{23}\left(\theta_{23}, \delta\right)$ rotation 4

$2.3 U_{13}(\phi)$ rotation 5

$2.4 U_{12}(\psi)$ rotation 6

2.5 Remarks 7

3 Perturbation expansion $\quad 9$

3.1 Corrections to the eigenvalues 9

$\begin{array}{lll}3.2 & \text { Corrections to the eigenvectors } & 10\end{array}$

4 Oscillation probabilities $\quad 11$

$\begin{array}{lll}4.1 & \text { The zeroth order probabilities } & 12\end{array}$

$\begin{array}{ll}4.2 \text { The first order probabilities } & 12\end{array}$

4.3 The second order probabilities 13

$\begin{array}{lll}4.4 & \text { Precision of the perturbation expansion } & 15\end{array}$

$\begin{array}{llr}5 & \text { Conclusions } & 16\end{array}$

$\begin{array}{ll}\text { A Technical details } & 17\end{array}$

$\begin{array}{ll}\text { A.1 Generalized approach to diagonalization } & 17\end{array}$

$\begin{array}{lll}\text { A.2 Useful identities } & 18\end{array}$

$\begin{array}{llr}\text { A.3 Limits } & 18\end{array}$

$\begin{array}{ll}\text { A.4 Characteristic equation } & 19\end{array}$

$\begin{array}{ll}\text { A.5 Unitarity of the } W \text { matrix } & 19\end{array}$

A.6 $V$-matrix, $S$-matrix comparison 20

$\begin{array}{lll}\text { A.7 } & C P \text { violating term } & 20\end{array}$

\section{Introduction}

Neutrino oscillation based on the standard three flavor scheme provides the best possible theoretical paradigm which can describe most of the experimental results obtained in the atmospheric, solar, reactor, and the accelerator neutrino experiments. In matter, the propogation of neutrinos is significantly modified by the Wolfenstein matter effect [1]. The theoretical derivation and understanding of the neutrino oscillation probabilities in matter have been pursued by various means. The exact expressions of the eigenvalues, mixing angles, and the oscillation probabilities have been obtained [2-4], albeit under the assumption of uniform matter density. But the resulting expressions of the oscillation 
probabilities are way too complex to facilitate understanding of the structure of the three flavor neutrino oscillations. For this reason, analytic approaches to the phenomena are mostly based on variety of perturbative frameworks. For a comprehensive treatment of neutrino oscillation in the matter, see ref. [5]. Analytic expressions for neutrino oscillations in arbitrary matter densities has also been considered, but even more simplifying arguments must be made [6].

What is the appropriate expansion parameter in such a perturbative framework? We now know that $\sin \theta_{13}$, once used as the expansion parameter (there are an enormous number of references, see e.g., [7]), is not so small, $\sin \theta_{13} \simeq 0.15$. Moreover, expansion around $\sin \theta_{13}=0$ misses the physics of the resonance which exists at an energy around $E \sim 10 \mathrm{GeV}$ for earth densities. Therefore, in the environments in which the matter effect is comparable to the vacuum mixing effect, the only available small expansion parameter known to us is the ratio of the solar-scale $\Delta m_{\odot}^{2}$ to the atmospheric-scale $\Delta m_{\oplus}^{2}, \Delta m_{\odot}^{2} / \Delta m_{\oplus}^{2} \simeq 0.03$. This framework was examined in the past, to our knowledge in refs. [7-10].

Recently, two of us, see [11], presented a new perturbative framework for neutrino oscillation in matter using a modified $\Delta m_{\odot}^{2} / \Delta m_{\oplus}^{2}$ expansion. We identified a unique $\Delta m_{\oplus}^{2}$ that absorb certain "first-order" terms into the "zeroth-order" Hamiltonian. The resulting expansion parameter,

$$
\epsilon \equiv \Delta m_{21}^{2} / \Delta m_{e e}^{2} \quad \text { where } \quad \Delta m_{e e}^{2} \equiv \Delta m_{31}^{2}-\sin ^{2} \theta_{12} \Delta m_{21}^{2},
$$

multiplies a particularly simple perturbing Hamiltonian with zero diagonal entries. This re-organization of the perturbation expansion lead to simple and compact oscillation probabilities in all channels. The $\nu_{e}$ disappearance channel is particularly simple, being of a pure two flavor form.

As was noted in [11], this new perturbation expansion, while valid in most of the baseline, $L$, divided by neutrino energy, $E$, versus matter potential plane, has issues around vacuum values for the matter potential at large values of $L / E$. These issues are caused by the crossing of two of the eigenvalues of the new zeroth order Hamiltonian at the solar resonance. In this paper, we solve these issues by performing an additional rotation of the neutrino basis in matter by introducing an additional matter mixing angle which is identical to $\theta_{12}$ in vacuum. With this extra rotation, the new eigenvalues of the unperturbed Hamiltonian do not cross and the perturbing Hamiltonian remains non-diagonal and is multiplied by an additional factor which is always less than unity and is zero in vacuum. With this additional rotation our perturbative expansion is valid in the full $L / E$ versus matter potential plane and the zeroth order gives the exact result in vacuum.

The sectional plan of this paper is as follows: in section 2 we describe in detail the sequence of rotations of the neutrino basis that leads us to the simple Hamiltonian that will be used in the perturbative expansion. The zeroth order eigenvalues and mixing matrix are given in this section. Then, in section, 3 we explicitly calculate the first and second order corrections for both the eigenvalues and the mixing matrix. In section 4 , we give compact analytic expressions for $\nu_{e}$ and $\nu_{\mu}$ disappearance channels as well as $\nu_{\mu} \rightarrow \nu_{e}$ appearance channel at both zeroth and first order in our perturbative expansion. All other channels can by obtained by unitarity. Here we discuss the precision of the perturbative 
treatment. Finally, in section 5 there is a conclusion. A number of technical details are contained in the appendices, see A. We have also published the new $\mathrm{Nu}$-Pert code used in this paper online. ${ }^{1}$

\section{Rotations of the neutrino basis and the Hamiltonian}

In this section we perform a sequence of rotations on the neutrino basis and the corresponding Hamiltonian such that the following conditions are satisfied:

- The diagonal elements of the rotated Hamiltonian are excellent approximations to the eigenvalues of the exact Hamiltonian and do not cross for any values of the matter potential. These diagonal elements will form our $H_{0}$.

- The size of non-diagonal elements are controlled by our small parameter, $\epsilon^{\prime}$, which vanishes in vacuum. The non-diagonal elements will form our perturbing Hamiltonian, $H_{1}$.

The first two of these rotations are identical to the rotations performed in [11], while the last rotation is needed to deal with the remaining eigenvalue crossing at the solar resonance. With these three rotations the resulting Hamiltonian satisfies the conditions above and leads us to a rapidly converging perturbative expansion for the oscillation probabilities that covers all of the $L / E$ versus matter potential plane.

\subsection{Overview}

Neutrino evolution in matter is governed by a Schrödinger like equation

$$
i \frac{\partial}{\partial x}|\nu\rangle=H|\nu\rangle
$$

where in the flavor basis

$$
\begin{aligned}
|\nu\rangle & =\left(\begin{array}{c}
\nu_{e} \\
\nu_{\mu} \\
\nu_{\tau}
\end{array}\right), \\
H & =\frac{1}{2 E}\left[U_{\mathrm{MNS}} \operatorname{diag}\left(0, \Delta m_{21}^{2}, \Delta m_{31}^{2}\right) U_{\mathrm{MNS}}^{\dagger}+\operatorname{diag}(a(x), 0,0)\right] .
\end{aligned}
$$

$U_{\mathrm{MNS}}$ is the lepton mixing matrix in vacuum, given by $U_{\mathrm{MNS}} \equiv U_{23}\left(\theta_{23}, \delta\right) U_{13}\left(\theta_{13}\right) U_{12}\left(\theta_{12}\right)$ with $^{2}$

$$
\begin{aligned}
& U_{12}(\psi) \equiv\left(\begin{array}{ccc}
c_{\psi} & s_{\psi} & \\
-s_{\psi} & c_{\psi} & \\
& & 1
\end{array}\right), \quad U_{13}(\phi) \equiv\left(\begin{array}{ccc}
c_{\phi} & & s_{\phi} \\
& 1 & \\
-s_{\phi} & & c_{\phi}
\end{array}\right) \\
& U_{23}\left(\theta_{23}, \delta\right) \equiv\left(\begin{array}{ccc}
1 & & \\
& c_{23} & s_{23} e^{i \delta} \\
-s_{23} e^{-i \delta} & c_{23}
\end{array}\right) \text {, }
\end{aligned}
$$

\footnotetext{
${ }^{1}$ See https://github.com/PeterDenton/Nu-Pert.

${ }^{2}$ The PDG form of $U_{\mathrm{MNS}}$ is obtained from our $U_{\mathrm{MNS}}$ by multiplying the $3 \mathrm{rd}$ row by $e^{i \delta}$ and the $3 \mathrm{rd}$ column by $e^{-i \delta}$ i.e. by rephasing $\nu_{\tau}$ and $\nu_{3}$. The shorthand notation $c_{\theta}=\cos \theta$ and $s_{\theta}=\sin \theta$ is used throughout this paper.
} 
and the matter potential, assumed to be constant, is given by

$$
a \equiv 2 \sqrt{2} G_{F} N_{e} E \approx 1.52 \times 10^{-4}\left(\frac{Y_{e} \rho}{\mathrm{g} \cdot \mathrm{cm}^{-3}}\right)\left(\frac{E}{\mathrm{GeV}}\right) \mathrm{eV}^{2} .
$$

We will perform a sequence of rotations on the flavor basis by multiplying the left and right hand side of eq. (2.1) by an appropriate unitary matrix, $U^{\dagger}$ and inserting unity $\left(U U^{\dagger}\right)$ between $H$ and $|\nu\rangle$. These rotations are chosen such that the final resulting Hamiltonian satisfies the following properties: the diagonal elements are an excellent approximations to the exact eigenvalues and the size of off-diagonal elements are controlled by a small parameter (ratio of the $\Delta m^{2}$ 's) and are identically zero in vacuum.

The sequence of rotations applied to the eigenstates is performed in the following order

$$
\begin{aligned}
|\nu\rangle \rightarrow|\tilde{\nu}\rangle & =U_{23}^{\dagger}\left(\theta_{23}, \delta\right)|\nu\rangle \\
\rightarrow|\hat{\nu}\rangle & =U_{13}^{\dagger}(\phi) U_{23}^{\dagger}\left(\theta_{23}, \delta\right)|\nu\rangle \\
\rightarrow|\check{\nu}\rangle & =U_{12}^{\dagger}(\psi) U_{13}^{\dagger}(\phi) U_{23}^{\dagger}\left(\theta_{23}, \delta\right)|\nu\rangle,
\end{aligned}
$$

with the corresponding Hamiltonians

$$
\begin{aligned}
H & \rightarrow \tilde{H}=U_{23}^{\dagger}\left(\theta_{23}, \delta\right) H U_{23}\left(\theta_{23}, \delta\right) \\
& \rightarrow \hat{H}=U_{13}^{\dagger}(\phi) U_{23}^{\dagger}\left(\theta_{23}, \delta\right) H U_{23}\left(\theta_{23}, \delta\right) U_{13}(\phi) \\
& \rightarrow \check{H}=U_{12}^{\dagger}(\psi) U_{13}^{\dagger}(\phi) U_{23}^{\dagger}\left(\theta_{23}, \delta\right) H U_{23}\left(\theta_{23}, \delta\right) U_{13}(\phi) U_{12}(\psi)
\end{aligned}
$$

The first rotation undoes the $\theta_{23}-\delta$ rotation, whereas the $\phi$ followed by $\psi$ rotations are matter analogues to the vacuum $\theta_{13}$ and $\theta_{12}$ rotations, respectively. In vacuum, the final Schrödinger equation is just the trivial mass eigenstate evolution equation.

\section{$2.2 U_{23}\left(\theta_{23}, \delta\right)$ rotation}

After the $U_{23}\left(\theta_{23}, \delta\right)$ rotation, the neutrino basis is

$$
|\tilde{\nu}\rangle=U_{23}^{\dagger}\left(\theta_{23}, \delta\right)|\nu\rangle
$$

and the Hamiltonian is given by

$$
\begin{aligned}
\tilde{H}= & U_{23}^{\dagger}\left(\theta_{23}, \delta\right) H U_{23}\left(\theta_{23}, \delta\right) \\
= & \frac{1}{2 E}\left[U_{13}\left(\theta_{13}\right) U_{12}\left(\theta_{12}\right) \operatorname{diag}\left(0, \Delta m_{21}^{2}, \Delta m_{31}^{2}\right) U_{12}^{\dagger}\left(\theta_{12}\right) U_{13}^{\dagger}\left(\theta_{13}\right)\right. \\
& +\operatorname{diag}(a, 0,0)] .
\end{aligned}
$$

As was shown in [11], the Hamiltonian, $\tilde{H}$, is most simple written in terms of a renormalized atmospheric $\Delta m^{2}$,

$$
\Delta m_{e e}^{2} \equiv \Delta m_{31}^{2}-s_{12}^{2} \Delta m_{21}^{2},
$$

as defined in $[12,13]$, and the ratio of the $\Delta m^{2}$ 's

$$
\epsilon \equiv \Delta m_{21}^{2} / \Delta m_{e e}^{2} .
$$


In terms of the $|a| \rightarrow \infty$ eigenvalues

$$
\begin{aligned}
\lambda_{a} & =a+\left(s_{13}^{2}+\epsilon s_{12}^{2}\right) \Delta m_{e e}^{2}, \\
\lambda_{b} & =\epsilon c_{12}^{2} \Delta m_{e e}^{2}, \\
\lambda_{c} & =\left(c_{13}^{2}+\epsilon s_{12}^{2}\right) \Delta m_{e e}^{2},
\end{aligned}
$$

the exact Hamiltonian is simple given by $^{3}$

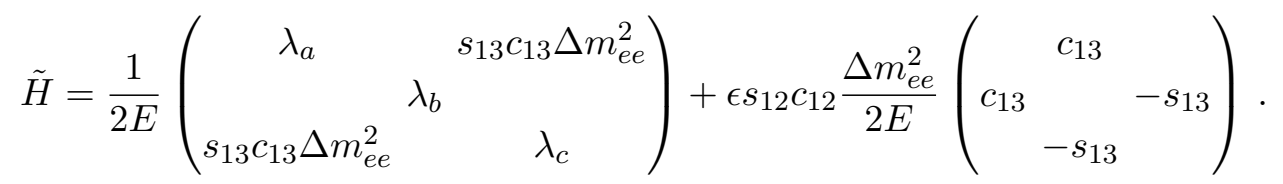

Note that $\tilde{H}$ is real and does not depend on $\theta_{23}$ or $\delta$.

\section{$2.3 \quad U_{13}(\phi)$ rotation}

Since $s_{13} \sim \mathcal{O}(\sqrt{\epsilon})$, it is natural to diagonalize the (1-3) sector next, using $U_{13}(\phi)$, again see [11]. After this rotation the neutrino basis is

$$
|\hat{\nu}\rangle=U_{13}^{\dagger}(\phi)|\tilde{\nu}\rangle=U_{13}^{\dagger}(\phi) U_{23}^{\dagger}\left(\theta_{23}, \delta\right)|\nu\rangle,
$$

and the Hamiltonian is given by

$$
\begin{aligned}
& \hat{H}=U_{13}^{\dagger}(\phi) \tilde{H} U_{13}(\phi) \\
& =\frac{1}{2 E}\left(\begin{array}{ccc}
\lambda_{-} & & \\
& \lambda_{0} & \\
& & \lambda_{+}
\end{array}\right)+\epsilon c_{12} s_{12} \frac{\Delta m_{e e}^{2}}{2 E}\left(\begin{array}{ccc} 
& c_{\left(\phi-\theta_{13}\right)} & \\
c_{\left(\phi-\theta_{13}\right)} & & s_{\left(\phi-\theta_{13}\right)} \\
& & s_{\left(\phi-\theta_{13}\right)}
\end{array}\right) \text {, }
\end{aligned}
$$

where

$$
\begin{aligned}
\lambda_{\mp} & =\frac{1}{2}\left[\left(\lambda_{a}+\lambda_{c}\right) \mp \operatorname{sign}\left(\Delta m_{e e}^{2}\right) \sqrt{\left(\lambda_{c}-\lambda_{a}\right)^{2}+4\left(s_{13} c_{13} \Delta m_{e e}^{2}\right)^{2}}\right], \\
\lambda_{0} & =\lambda_{b}=\epsilon c_{12}^{2} \Delta m_{e e}^{2},
\end{aligned}
$$

which is identical to eq. 3.1 of [11]. Also $c_{\left(\phi-\theta_{13}\right)} \equiv \cos \left(\phi-\theta_{13}\right)$ and $s_{\left(\phi-\theta_{13}\right)} \equiv \sin \left(\phi-\theta_{13}\right)$.

The angle, $\phi$, that achieves this diagonalization of the (1-3) sub-matrix (see appendix A.1), satisfies

$$
\lambda_{a}=c_{\phi}^{2} \lambda_{-}+s_{\phi}^{2} \lambda_{+}, \quad \lambda_{c}=s_{\phi}^{2} \lambda_{-}+c_{\phi}^{2} \lambda_{+}, \quad \text { and } \quad s_{\phi} c_{\phi}=\frac{s_{13} c_{13} \Delta m_{e e}^{2}}{\lambda_{+}-\lambda_{-}}
$$

from which it is easy to derive

$$
\begin{aligned}
c_{\phi}^{2}-s_{\phi}^{2} & =\frac{\lambda_{c}-\lambda_{a}}{\lambda_{+}-\lambda_{-}}, \\
s_{\phi} & =\sqrt{\frac{\lambda_{+}-\lambda_{c}}{\lambda_{+}-\lambda_{-}}}, \quad c_{\phi}=\sqrt{\frac{\lambda_{c}-\lambda_{-}}{\lambda_{+}-\lambda_{-}}} .
\end{aligned}
$$

\footnotetext{
${ }^{3}$ One can use $\tilde{H}$ to do a perturbative expansion, such that it is simple to recover the $\nu_{\mu} \rightarrow \nu_{e}$ appearance probability of Cervera et al., [7] at first order.
} 
The Hamiltonian given in eq. (2.15) was used to derive simple, compact and accurate oscillation probabilities for a wide range of the $L / E$ versus $\rho E$ plane, see [11]. However, as was noted in that paper, there is a region of this plane for which a perturbation theory based on $\hat{H}$ is insufficient to describe the physics accurately. This region is small $\rho E$ and large $L / E$ given by

$$
|a|<\frac{1}{3} \Delta m_{e e}^{2} \quad \text { and } \quad L / E>\frac{4 \pi}{\Delta m_{e e}^{2}} .
$$

To address this region of the $L / E$ versus $\rho E$ plane, we perform one further rotation on the Hamiltonian. This rotation removes the degeneracy of the zeroth order eigenvalues at the solar resonance when $\lambda_{-}=\lambda_{0}$. This is performed in the next subsection.

\section{$2.4 U_{12}(\psi)$ rotation}

Since $\lambda_{-}$and $\lambda_{0}$ cross at the solar resonance, $a \approx \epsilon \Delta m_{e e}^{2} \cos 2 \theta_{12} / \cos ^{2} \theta_{13}$, to describe the physics near this degeneracy we need to diagonalize the (1-2) submatrix of $\hat{H}$, using $U_{12}(\psi)$. The new neutrino basis is

$$
|\check{\nu}\rangle=U_{12}^{\dagger}(\psi)|\hat{\nu}\rangle=U_{12}^{\dagger}(\psi) U_{13}^{\dagger}(\phi) U_{23}^{\dagger}\left(\theta_{23}, \delta\right)|\nu\rangle .
$$

The resulting Hamiltonian, split into a zeroth order Hamiltonian and a perturbing Hamiltonian, is given by

$$
\check{H}=U_{12}^{\dagger}(\psi) \hat{H} U_{12}(\psi)=\check{H}_{0}+\check{H}_{1},
$$

where

$$
\begin{aligned}
& \check{H}_{0}=\frac{1}{2 E}\left(\begin{array}{lll}
\lambda_{1} & & \\
& \lambda_{2} & \\
& & \lambda_{3}
\end{array}\right), \\
& \check{H}_{1}=\epsilon s_{\left(\phi-\theta_{13}\right)} s_{12} c_{12} \frac{\Delta m_{e e}^{2}}{2 E}\left(\begin{array}{rr} 
& -s_{\psi} \\
& c_{\psi} \\
-s_{\psi} & c_{\psi}
\end{array}\right) .
\end{aligned}
$$

The diagonal elements of the zeroth order Hamiltonian are

$$
\begin{aligned}
\lambda_{1,2} & =\frac{1}{2}\left[\left(\lambda_{0}+\lambda_{-}\right) \mp \sqrt{\left(\lambda_{0}-\lambda_{-}\right)^{2}+4\left(\epsilon c_{\left(\phi-\theta_{13}\right)} c_{12} s_{12} \Delta m_{e e}^{2}\right)^{2}}\right], \\
\lambda_{3} & =\lambda_{+} .
\end{aligned}
$$

The angle, $\psi$, that achieves this diagonalization of the (1-2) sub-matrix of $\hat{H}$ (see appendix A.1), satisfies

$$
\begin{aligned}
\lambda_{-} & =c_{\psi}^{2} \lambda_{1}+s_{\psi}^{2} \lambda_{2}, \quad \lambda_{0}=s_{\psi}^{2} \lambda_{1}+c_{\psi}^{2} \lambda_{2}, \\
s_{\psi} c_{\psi} & =\frac{\epsilon c_{\left(\phi-\theta_{13}\right)} s_{12} c_{12} \Delta m_{e e}^{2}}{\Delta \lambda_{21}}
\end{aligned}
$$

where we introduce the useful shorthand notation,

$$
\Delta \lambda_{i j} \equiv \lambda_{i}-\lambda_{j}
$$


It is easy to derive that ${ }^{4}$

$$
\begin{aligned}
c_{\psi}^{2}-s_{\psi}^{2} & =\frac{\lambda_{0}-\lambda_{-}}{\Delta \lambda_{21}}, \\
\text { and } s_{\psi}=\sqrt{\frac{\lambda_{2}-\lambda_{0}}{\Delta \lambda_{21}}}, \quad c_{\psi} & =\operatorname{sign}\left(\Delta \lambda_{21}\right) \sqrt{\frac{\lambda_{0}-\lambda_{1}}{\Delta \lambda_{21}}} .
\end{aligned}
$$

Figure 1 shows $\phi$ and $\psi$ as functions of the matter potential as well as the eigenvalues of $\check{H}$ for both the normal ordering (NO) and the inverted ordering (IO). Several additional useful identities used in the calculations throughout this paper are listed in appendix A.2.

\subsection{Remarks}

A number of summarizing and useful comments are warranted at this point.

- The neutrino basis that will be used in our perturbation theory, $|\check{\nu}\rangle$ is related to the flavor basis, $|\nu\rangle$ by

where

$$
\left(\begin{array}{c}
\nu_{e} \\
\nu_{\mu} \\
\nu_{\tau}
\end{array}\right)=U_{\mathrm{MNS}}^{m}\left(\begin{array}{c}
\check{\nu}_{1} \\
\check{\nu}_{2} \\
\check{\nu}_{3}
\end{array}\right),
$$

$$
U_{\mathrm{MNS}}^{m} \equiv U_{23}\left(\theta_{23}, \delta\right) U_{13}(\phi) U_{12}(\psi) .
$$

- The Hamiltonian, eqs. (2.23) and (2.24), that will used as the basis for our perturbation theory is given by

$$
\check{H}=\left(U_{\mathrm{MNS}}^{m}\right)^{\dagger} H U_{\mathrm{MNS}}^{m}=\check{H}_{0}+\check{H}_{1},
$$

with the diagonal elements the zeroth order Hamiltonian and the off-diagonal elements the perturbing Hamiltonian. While the $\lambda_{a, b, c}$ eigenvalues cross twice and the $\lambda_{-, 0,+}$ eigenvalues cross once, the new $\lambda_{1,2,3}$ eigenvalues do not cross, see figure 1 , which allows for the perturbation theory to be well defined everywhere.

- The size of the perturbing Hamiltonian, $\check{H}_{1}$, is controlled by the parameter

$$
\begin{aligned}
\epsilon^{\prime} & \equiv \epsilon s_{\left(\phi-\theta_{13}\right)} s_{12} c_{12} \\
& =s_{\left(\phi-\theta_{13}\right)} s_{12} c_{12} \frac{\Delta m_{21}^{2}}{\Delta m_{e e}^{2}},
\end{aligned}
$$

which is never larger than $1.4 \%$.

- In vacuum,

$$
s_{\left(\phi-\theta_{13}\right)}=0,
$$

so that the zeroth order Hamiltonian gives the exact result. Also, in the limit where $a \rightarrow-\infty$ for NO or $a \rightarrow+\infty$ for IO $s_{\left(\phi-\theta_{13}\right)} \rightarrow-s_{13}$ which is of $\mathcal{O}(\sqrt{\epsilon})$. Whereas for $a \rightarrow+\infty$ for NO or $a \rightarrow-\infty$ for IO $s_{\left(\phi-\theta_{13}\right)} \rightarrow c_{13} \sim 1$, see figure 2 .

- Since perturbing Hamiltonian, $\check{H}_{1}$, has only non-diagonal entries the first order correction to the eigenvalues are zero. The diagonal elements multiplied by $2 E$ are, to an excellent approximation, the mass squares of the neutrinos in matter.

\footnotetext{
${ }^{4}$ Given the definition of $\lambda_{1,2}$ in eq. (2.25), the sign term in from of $c_{\psi}$ is not necessary, but will become necessary when we discuss the $\lambda_{1} \leftrightarrow \lambda_{2}$ interchange symmetry.
} 

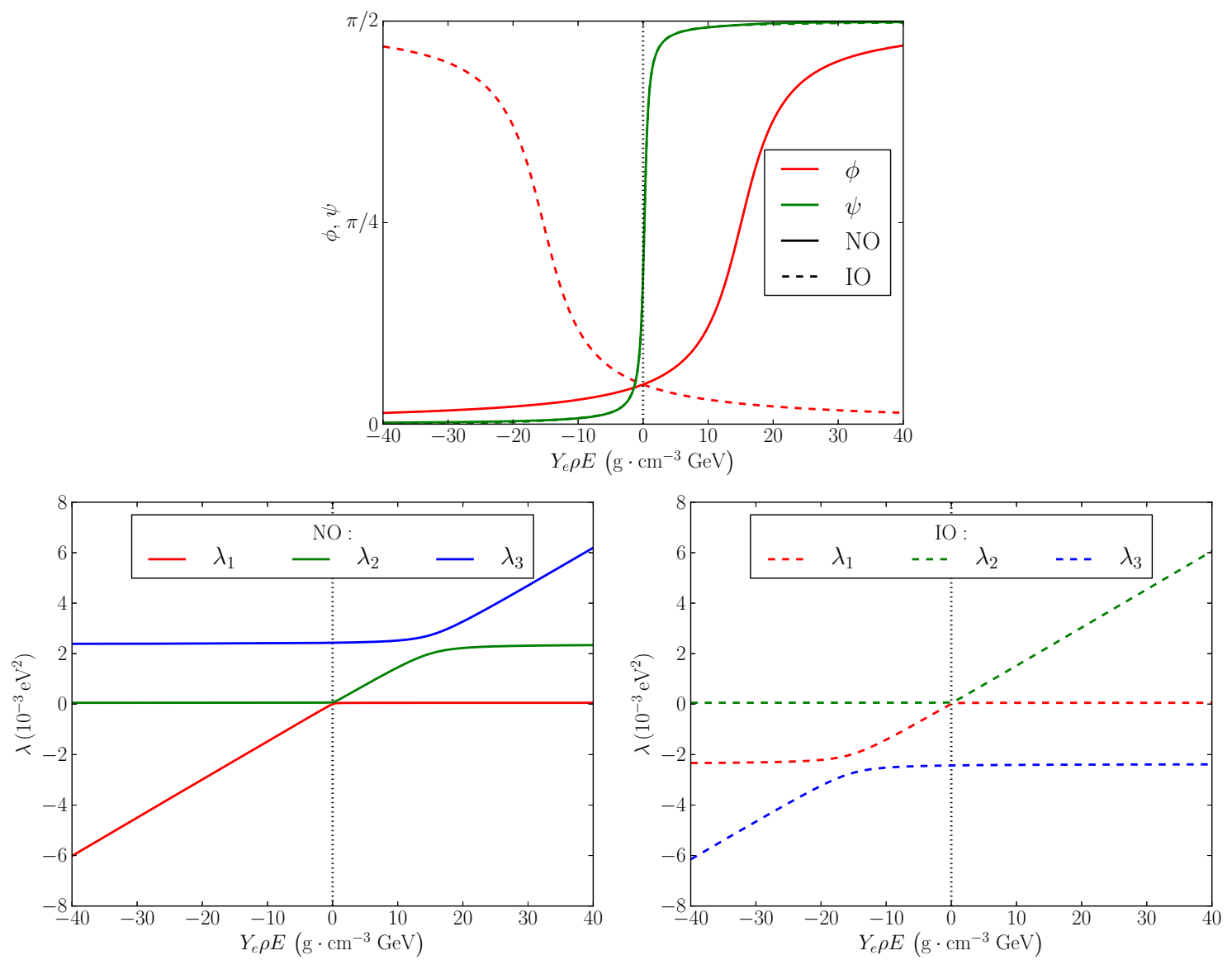

Figure 1. The upper figure shows the angles, $\phi$ and $\psi$, as a function of the matter potential for both NO and IO. $\phi$ and $\psi$ are the mixing angles $\theta_{13}$ and $\theta_{12}$ in matter respectively. For $\psi$, the curves for the two mass ordering are nearly identical. The two lower figures show the eigenvalues to zeroth order, $\lambda_{1,2,3}$, in matter as a function of the matter potential for NO and for IO. For all our figures, $Y_{e} \rho E \geq 0$ is for neutrinos and $Y_{e} \rho E \leq 0$ for antineutrinos.

- There is a very useful interchange symmetry involving $\lambda_{1,2}$ and $\psi$. The Hamiltonian is invariant under the pair of transformations $\lambda_{1} \leftrightarrow \lambda_{2}$ and $\psi \rightarrow \psi \pm \pi / 2$. Our expressions for $s_{\psi}$ and $c_{\psi}$, see eq. (2.30), satisfy this interchange symmetry with the + in front of the $\pi / 2$. Since the transition probabilities always have an even number of $\psi$ trig functions, this interchange symmetry can be simply expressed as

$$
\lambda_{1} \leftrightarrow \lambda_{2}, \quad c_{\psi}^{2} \leftrightarrow s_{\psi}^{2}, \quad \text { and } \quad c_{\psi} s_{\psi} \leftrightarrow-c_{\psi} s_{\psi}
$$

In the rest of this paper we call this the $\lambda_{1,2}-\psi$ interchange symmetry.

- An antineutrino with energy $E$ is equivalent to a neutrino with energy $-E$.

- The values of all of the eigenvalues in vacuum and for $a \rightarrow \pm \infty$ are shown in appendix A.3. 


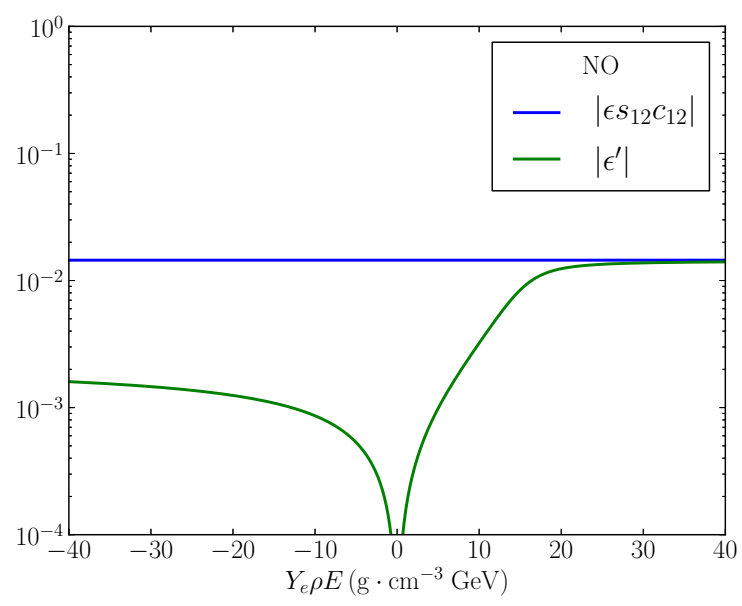

Figure 2. The absolute value of the various expansion parameters as a function of the matter potential. $\epsilon s_{12} c_{12} \equiv s_{12} c_{12} \Delta m_{21}^{2} / \Delta m_{e e}^{2}$ is the expansion parameter from [11] and $\epsilon^{\prime} \equiv s_{\left(\phi-\theta_{13}\right)} s_{12} c_{12} \Delta m_{21}^{2} / \Delta m_{e e}^{2}$ is the expansion parameter of this paper, see eqs. (2.11) and (2.34). The asymptotic value of $\left|\epsilon^{\prime}\right|$ as $E \rightarrow-\infty$ is $\left|\epsilon c_{12} s_{12} s_{13}\right| \approx 2.2 \times 10^{-3}$ and as $E \rightarrow \infty$ is $\left|\epsilon c_{12} s_{12} c_{13}\right| \approx 1.4 \times 10^{-2}$. The NO is shown here, the IO is the same with $Y_{e} \rho E \rightarrow-Y_{e} \rho E$.

\section{Perturbation expansion}

To calculate the neutrino oscillation probabilities at zeroth order, all that is needed is eigenvalues and mixing matrix,

$$
\lambda_{1,2,3} \text { and } U_{\mathrm{MNS}}^{m},
$$

given by eq. (2.25) and eq. (2.32) respectively. For higher order calculations we need not only the corrections to the eigenvalues but also the corrections to the mixing matrix. In this section we first given the corrections to the eigenvalues at both first and second order in our expansion parameter, $\epsilon^{\prime}$. This is followed by the corrections to the same order for the mixing matrix. Note that all corrections to both the eigenvalues and the mixing matrix vanish in vacuum as our expansion parameter is zero in vacuum, i.e. the zero order oscillation probabilities are exact in vacuum.

\subsection{Corrections to the eigenvalues}

Since the diagonal terms of $\check{H}_{1}=0$ by construction, the first order corrections to the eigenvalues are exactly zero, since

$$
\lambda_{i}^{(1)}=2 E\left(\check{H}_{1}\right)_{i i}=0 .
$$

The second order corrections to the eigenvalues are given by ${ }^{5}$

$$
\lambda_{i}^{(2)}=\sum_{k \neq i} \frac{\left[2 E\left(\check{H}_{1}\right)_{i k}\right]^{2}}{\Delta \lambda_{i k}} .
$$

\footnotetext{
${ }^{5}$ Eq. (3.2) explicitly shows why the level crossing of two of the eigenvalues $\left(\lambda_{-}, \lambda_{0}\right)$ causes problems for higher orders in the perturbation theory.
} 
Using $\check{H}_{1}$ from eq. (2.24), we see that the corrections are

$$
\begin{aligned}
& \lambda_{1}^{(2)}=-\left(\epsilon^{\prime} \Delta m_{e e}^{2}\right)^{2} \frac{s_{\psi}^{2}}{\Delta \lambda_{31}}, \\
& \lambda_{2}^{(2)}=-\left(\epsilon^{\prime} \Delta m_{e e}^{2}\right)^{2} \frac{c_{\psi}^{2}}{\Delta \lambda_{32}}, \\
& \lambda_{3}^{(2)}=\left(\epsilon^{\prime} \Delta m_{e e}^{2}\right)^{2}\left(\frac{s_{\psi}^{2}}{\Delta \lambda_{31}}+\frac{c_{\psi}^{2}}{\Delta \lambda_{32}}\right) .
\end{aligned}
$$

We verified that the eigenvalues satisfy the characteristic equation to second order, see appendix A.4. The eigenvalues are correct at zeroth order to a fractional precision of about $10^{-4}$ or better, and through second order to a precision of $10^{-8}$ or better. In fact, the precision of $\lambda_{1}+\lambda_{1}^{(1)}+\lambda_{1}^{(2)}$ for $\operatorname{sign}\left(\Delta m_{e e}^{2}\right) Y_{e} \rho E<0$ is completely saturated by the limits of double precision computer calculations.

\subsection{Corrections to the eigenvectors}

Here we present the corrections to the eigenvectors which allows us to calculate the transition probabilities to second order. Higher orders can be easily calculated by continuing this approach in a straightforward fashion. This was called the $V$-matrix approach in [14].

First, we relate the flavor eigenvectors to the zeroth order eigenvectors (no subscript) using $U_{\mathrm{MNS}}^{m}$, as in eq. (2.4),

$$
\left(\begin{array}{c}
\nu_{e} \\
\nu_{\mu} \\
\nu_{\tau}
\end{array}\right)=U_{\mathrm{MNS}}^{m}\left(\begin{array}{l}
\check{\nu}_{1} \\
\check{\nu}_{2} \\
\check{\nu}_{3}
\end{array}\right) .
$$

Next, the exact eigenvectors of $\check{H}$, labeled with subscript (ex), are related to the eigenvectors of $\breve{H}_{0}$ (the zeroth order eigenvectors) by a unitary matrix, which we call $W^{\dagger}$,

$$
\left(\begin{array}{l}
\check{\nu}_{1} \\
\check{\nu}_{2} \\
\check{\nu}_{3}
\end{array}\right)_{(\mathrm{ex})}=W^{\dagger}\left(\begin{array}{c}
\check{\nu}_{1} \\
\check{\nu}_{2} \\
\check{\nu}_{3}
\end{array}\right) \text {. }
$$

Combining the above gives,

$$
\left(\begin{array}{l}
\nu_{e} \\
\nu_{\mu} \\
\nu_{\tau}
\end{array}\right)=V\left(\begin{array}{l}
\check{\nu}_{1} \\
\check{\nu}_{2} \\
\check{\nu}_{3}
\end{array}\right)_{(\mathrm{ex})} \quad \text { where } \quad V \equiv U_{\mathrm{MNS}}^{m} W .
$$

The exact $V$ matrix transforms the exact eigenvectors of $\check{H}$ to the flavor basis. In vacuum $(a=0), U_{\mathrm{MNS}}^{m}=U_{\mathrm{MNS}}$ and $W=\mathbb{1}$, so $V=U_{\mathrm{MNS}}$ as expected.

Standard perturbation theory in $\check{H}_{1}$, which contains the small parameter $\epsilon^{\prime}$, can be used to calculate $W^{\dagger}$. Here we use a slightly modified perturbation theory to calculate $W$ directly. Expanding $W$ as a power series in $\epsilon^{\prime}$, we define

$$
W \equiv W_{0}+W_{1}+W_{2}+\mathcal{O}\left(\epsilon^{\prime 3}\right) .
$$

It is clear from eq. (3.5) that $W_{0}=\mathbb{1}$. 
The first order correction to the $W$ matrix is given by

$$
\begin{aligned}
\left(W_{1}\right)_{i j} & =\left\{\begin{array}{lll}
0 & i=j \\
-\frac{2 E\left(\check{H}_{1}\right)_{i j}}{\Delta \lambda_{i j}} & i \neq j
\end{array},\right. \text { thus } \\
W_{1} & =\epsilon^{\prime} \Delta m_{e e}^{2}\left(\begin{array}{rrr}
\frac{s_{\psi}}{\Delta \lambda_{31}} \\
\frac{s_{\psi}}{\Delta \lambda_{31}} & -\frac{c_{\psi}}{\Delta \lambda_{32}} &
\end{array}\right) .
\end{aligned}
$$

The second order correction, after using the facts that $\check{H}_{1}$ is symmetric and has no diagonal elements, eq. (2.24), is

$$
\begin{aligned}
\left(W_{2}\right)_{i j} & =\left\{\begin{array}{ll}
-\frac{1}{2} \sum_{k \neq i} \frac{\left[2 E\left(\check{H}_{1}\right)_{i k}\right]^{2}}{\left(\Delta \lambda_{i k}\right)^{2}} & i=j \\
\frac{1}{\Delta \lambda_{i j}} \sum_{k \neq i, k \neq j} \frac{2 E\left(\check{H}_{1}\right)_{i k} 2 E\left(\check{H}_{1}\right)_{k j}}{\Delta \lambda_{k j}} & i \neq j
\end{array},\right. \text { thus } \\
W_{2} & =-\epsilon^{\prime 2} \frac{\left(\Delta m_{e e}^{2}\right)^{2}}{2}\left(\begin{array}{lll}
\frac{s_{\psi}^{2}}{\left(\Delta \lambda_{31}\right)^{2}} & -\frac{s_{2 \psi}}{\Delta \lambda_{32} \Delta \lambda_{21}} \\
\frac{s_{2 \psi}}{\Delta \lambda_{31} \Delta \lambda_{21}} & \frac{c_{\psi}^{2}}{\left(\Delta \lambda_{32}\right)^{2}} \\
& {\left[\frac{c_{\psi}^{2}}{\left(\Delta \lambda_{32}\right)^{2}}+\frac{s_{\psi}^{2}}{\left(\Delta \lambda_{31}\right)^{2}}\right]}
\end{array}\right) .
\end{aligned}
$$

This series can be continued to reach arbitrary precision. However, we have found that second order provides more than sufficient precision.

In summary the matrix relating the zeroth order eigenvalues of $\check{H}_{0}$ to the flavor basis is given by

$$
V=U_{\mathrm{MNS}}^{m} W=U_{23}\left(\theta_{23}, \delta\right) U_{13}(\phi) U_{12}(\psi)\left(\mathbb{1}+W_{1}+W_{2}\right),
$$

to second order in $\epsilon^{\prime}$. Demonstration of the unitary nature of $V$, to the appropriate order, is given in appendix A.5. With the eigenvalues and eigenvectors determined to second order we can now calculate the neutrino oscillation probabilities.

\section{Oscillation probabilities}

In vacuum and in matter with constant density, it is well known that the neutrino oscillation probabilities for $\nu_{\alpha} \rightarrow \nu_{\beta}$ for three-flavor mixing $(i, j=1,2,3)$ can be written in the following form ${ }^{6}$

$$
\begin{aligned}
P\left(\nu_{\alpha} \rightarrow \nu_{\beta}\right)= & \left|\sum_{i=1}^{3} V_{\alpha i}^{*} V_{\beta i} e^{-i \frac{\lambda_{i}^{(\mathrm{ex})} L}{2 E}}\right|^{2} \\
= & \delta^{\alpha \beta}+4 C_{21}^{\alpha \beta} \sin ^{2} \Delta_{21}+4 C_{31}^{\alpha \beta} \sin ^{2} \Delta_{31}+4 C_{32}^{\alpha \beta} \sin ^{2} \Delta_{32} \\
& +8 D^{\alpha \beta} \sin \Delta_{21} \sin \Delta_{31} \sin \Delta_{32}
\end{aligned}
$$

\footnotetext{
${ }^{6}$ The equivalence of the V-matrix method and the S-matrix method for calculating the oscillation probabilities is addressed in appendix A.6.
} 
where

$$
\begin{aligned}
C_{i j}^{\alpha \beta} & =-\Re\left[V_{\alpha i} V_{\beta i}^{*} V_{\alpha j}^{*} V_{\beta j}\right], \\
D^{\alpha \beta} & =\Im\left[V_{\alpha 1} V_{\beta 1}^{*} V_{\alpha 2}^{*} V_{\beta 2}\right], \\
\Delta_{i j} & \equiv \Delta \lambda_{i j}^{(\mathrm{ex})} L / 4 E,
\end{aligned}
$$

using the exact mixing matrix, $V_{\alpha i}$, and difference of the exact eigenvalues $\lambda_{i}^{(\mathrm{ex})}$. Both $V$ and $\lambda_{i}^{(\text {ex) }}$ s depend on the energy of the neutrino $E$, and the matter density $\rho$ but the baseline $L$, dependence only appears in $\Delta_{i j}$.

By unitarity

$$
\sum_{\beta} P\left(\nu_{\alpha} \rightarrow \nu_{\beta}\right)=1
$$

and using the fact that the $\sin ^{2}$ functions and the triple sine function are linearly independent functions of $\mathrm{L}$, as determined by their non-zero Wronskian, we have the following powerful statements,

$$
\sum_{\beta} C_{i j}^{\alpha \beta}=0, \quad \sum_{\beta} D^{\alpha \beta}=0
$$

Since $D^{\alpha \alpha}=0$, we also note that $D^{\alpha \beta}=-D^{\alpha \gamma}$ for $\alpha, \beta, \gamma$ all different. So, up to one overall sign, there is only one $D$ term for all channels.

To determine the oscillation probability to n-th order in our perturbative expansion we must evaluate $C, D$, and $\Delta \lambda_{i j}^{(\mathrm{ex})}$ to the $\mathrm{n}$-th order. We denote this perturbative expansion as follows

$$
\begin{aligned}
\Delta \lambda_{i j}^{(\mathrm{ex})} & =\Delta \lambda_{i j}+\Delta \lambda_{i j}^{(1)}+\Delta \lambda_{i j}^{(2)}+\ldots \\
C_{i j}^{\alpha \beta} & =\left(C_{i j}^{\alpha \beta}\right)^{(0)}+\left(C_{i j}^{\alpha \beta}\right)^{(1)}+\left(C_{i j}^{\alpha \beta}\right)^{(2)}+\ldots \\
D^{\alpha \beta} & =\left(D^{\alpha \beta}\right)^{(0)}+\left(D^{\alpha \beta}\right)^{(1)}+\left(D^{\alpha \beta}\right)^{(2)}+\ldots
\end{aligned}
$$

\subsection{The zeroth order probabilities}

At zeroth order the $\Delta \lambda$ 's are given by eq. (2.25) and the $C, D$ coefficients are the same as in vacuum with $\theta_{13}, \theta_{12}$ replaced with $\phi, \psi$ respectively, see eq. (3.10). Therefore

$$
\begin{aligned}
& \left(C_{i j}^{\alpha \beta}\right)^{(0)}=-\Re\left[U_{\alpha i} U_{\beta i}^{*} U_{\alpha j}^{*} U_{\beta j}\right], \\
& \left(D^{\alpha \beta}\right)^{(0)}=\Im\left[U_{\alpha 1} U_{\beta 1}^{*} U_{\alpha 2}^{*} U_{\beta 2}\right],
\end{aligned}
$$

where here the $U_{\alpha i}$ are elements of $U_{\text {MNS }}^{m}=U_{23}\left(\theta_{23}, \delta\right) U_{13}(\phi) U_{12}(\psi)$. In table 1 we give the zeroth order coefficients for $P\left(\nu_{e} \rightarrow \nu_{e}\right), P\left(\nu_{\mu} \rightarrow \nu_{e}\right)$, and $P\left(\nu_{\mu} \rightarrow \nu_{\mu}\right)$, from which all remaining transitions can be easily determined by unitarity. ${ }^{7}$

\subsection{The first order probabilities}

At first order the $\Delta \lambda$ 's are again given by eq. (2.25), since $\lambda_{i}^{(1)}=0$, see eq. (2.24), because the diagonal elements of $\check{H}_{1}$ are zero. The first order corrections to $C, D$ only have terms proportional to $\Delta \lambda_{31}^{-1}, \Delta \lambda_{32}^{-1}$. This comes from the form of $W_{1}$, eq. (3.8), which follows

\footnotetext{
${ }^{7}$ The $\nu_{\tau}$ channels can also be obtained from the corresponding $\nu_{\mu}$ channel by the following replacements $c_{23} \rightarrow-s_{23}$ and $s_{23} \rightarrow c_{23}$.
} 
from the position of the non-zero elements in $\check{H}_{1}$. In fact, all of the coefficients can be written in the following general form,

$$
\begin{aligned}
& \left(C_{21}^{\alpha \beta}\right)^{(1)}=\epsilon^{\prime} \Delta m_{e e}^{2}\left(\frac{F_{1}^{\alpha \beta}}{\Delta \lambda_{31}}+\frac{F_{2}^{\alpha \beta}}{\Delta \lambda_{32}}\right), \\
& \left(C_{31}^{\alpha \beta}\right)^{(1)}=\epsilon^{\prime} \Delta m_{e e}^{2}\left(\frac{F_{1}^{\alpha \beta}+G_{1}^{\alpha \beta}}{\Delta \lambda_{31}}-\frac{F_{2}^{\alpha \beta}}{\Delta \lambda_{32}}\right), \\
& \left(C_{32}^{\alpha \beta}\right)^{(1)}=\epsilon^{\prime} \Delta m_{e e}^{2}\left(-\frac{F_{1}^{\alpha \beta}}{\Delta \lambda_{31}}+\frac{F_{2}^{\alpha \beta}+G_{2}^{\alpha \beta}}{\Delta \lambda_{32}}\right), \\
& \left(D^{\alpha \beta}\right)^{(1)}=\epsilon^{\prime} \Delta m_{e e}^{2}\left(\frac{K_{1}^{\alpha \beta}}{\Delta \lambda_{31}}-\frac{K_{2}^{\alpha \beta}}{\Delta \lambda_{32}}\right),
\end{aligned}
$$

where the $F_{1,2}, G_{1,2}$ and $K_{1,2}$ are related by $\lambda_{1,2}, \psi$ interchange previously discussed. Thus only three modest expressions are required to describe the $C$ 's and $D$ coefficients to first order for each channel. The $F, G, K$ terms can be calculated from $U_{\mathrm{MNS}}^{m}$ by

$$
\begin{aligned}
& F_{1}^{\alpha \beta}=-s_{\psi} \Re\left[\left(U_{\alpha 1} U_{\beta 3}^{*}+U_{\alpha 3} U_{\beta 1}^{*}\right) U_{\alpha 2}^{*} U_{\beta 2}\right], \\
& G_{1}^{\alpha \beta}=-s_{\psi} \Re\left[\left(U_{\alpha 1} U_{\beta 3}^{*}+U_{\alpha 3} U_{\beta 1}^{*}\right)\left(2 U_{\alpha 3}^{*} U_{\beta 3}-\delta_{\alpha \beta}\right)\right], \\
& K_{1}^{\alpha \beta}=-s_{\psi} \mathcal{I}\left[\left(U_{\alpha 1} U_{\beta 3}^{*}+U_{\alpha 3} U_{\beta 1}^{*}\right) U_{\alpha 2}^{*} U_{\beta 2}\right] .
\end{aligned}
$$

$F$ and $G$ are even under the interchange of $\alpha$ and $\beta$ whereas $K$ is odd. Their explicit values are given in table 2 .

In the appearance channels the $C P$ violating term must be of the following form

$$
D= \pm s_{12} c_{12} s_{13} c_{13}^{2} s_{23} c_{23} \sin \delta \frac{\prod_{i>j} \Delta m_{i j}^{2}}{\prod_{i>j} \Delta \lambda_{i j}^{(\mathrm{ex})}},
$$

where in the denominator one needs the exact eigenvalues in matter. This is the NaumovHarrison-Scott identity, see refs. $[15,16]$. We have checked this identity to the appropriate order, see appendix A.7.

The $P\left(\nu_{\alpha} \rightarrow \beta\right)$ and $P\left(\bar{\nu}_{\alpha} \rightarrow \bar{\nu}_{\beta}\right)$ probabilities are related by $\delta \rightarrow-\delta$ and the $P\left(\nu_{\alpha} \rightarrow \nu_{\beta}\right)$ and $P\left(\nu_{\beta} \rightarrow \nu_{\alpha}\right)$ transition probabilities are related by $L \rightarrow-L$. From eq. (4.1), we see that the $D$ term is the only term odd in $L$. From tables 1 and 2, we see that the $D$ term is also the only one odd in $\delta$, confirming the CPT invariance of these equations. Moreover, all of the $D^{\alpha \beta}$ terms are the same order by order up to a coefficient of $-1,0,1$.

\subsection{The second order probabilities}

Although we have not expanded the second order oscillation probabilities analytically, the second order corrections to the eigenvalues, $\lambda_{i}^{(2)}$, as well as the second order corrections to the mixing matrix, $W_{2}$, have been used to calculate the oscillation probabilities to second order. The resulting oscillation probabilities are more than two orders of magnitude closer to the exact values than the first order probabilities. 


\begin{tabular}{|c|c|c|c|}
\hline$\nu_{\alpha} \rightarrow \nu_{\beta}$ & $\left(C_{31}^{\alpha \beta}\right)^{(0)}$ & $\left(C_{21}^{\alpha \beta}\right)^{(0)}$ & $\left(D^{\alpha \beta}\right)^{(0)}$ \\
\hline$\nu_{e} \rightarrow \nu_{e}$ & $-c_{\phi}^{2} s_{\phi}^{2} c_{\psi}^{2}$ & $-c_{\phi}^{4} s_{\psi}^{2} c_{\psi}^{2}$ & 0 \\
\hline$\nu_{\mu} \rightarrow \nu_{e}$ & $s_{\phi}^{2} c_{\phi}^{2} c_{\psi}^{2} s_{23}^{2}+J_{r}^{m} \cos \delta$ & $c_{\phi}^{2} s_{\psi}^{2} c_{\psi}^{2}\left(c_{23}^{2}-s_{\phi}^{2} s_{23}^{2}\right)+c_{2 \psi} J_{r}^{m} \cos \delta$ & $-J_{r}^{m} \sin \delta$ \\
\hline & $-c_{\phi}^{2} s_{23}^{2}\left(c_{23}^{2} s_{\psi}^{2}+s_{23}^{2} s_{\phi}^{2} c_{\psi}^{2}\right)$ & $-\left(c_{23}^{2} c_{\psi}^{2}+s_{23}^{2} s_{\phi}^{2} s_{\psi}^{2}\right)\left(c_{23}^{2} s_{\psi}^{2}+s_{23}^{2} s_{\phi}^{2} c_{\psi}^{2}\right)$ & \\
$\nu_{\mu} \rightarrow \nu_{\mu}$ & $-2 s_{23}^{2} J_{r}^{m} \cos \delta$ & $-2\left(c_{23}^{2}-s_{\phi}^{2} s_{23}^{2}\right) c_{2 \psi} J_{r r}^{m} \cos \delta+\left(2 J_{r r}^{m} \cos \delta\right)^{2}$ & 0 \\
\hline
\end{tabular}

Table 1. The zeroth order coefficients for $C_{i j}^{\alpha \beta}$ and $D^{\alpha \beta}$ using eq. (4.6). The angles in matter, $\phi, \psi$, are given in sections 2.3 and 2.4. We also define the singly and doubly reduced Jarlskog coefficients in matter as $J_{r}^{m} \equiv s_{\psi} c_{\psi} s_{\phi} c_{\phi}^{2} s_{23} c_{23}$ and $J_{r r}^{m} \equiv J_{r}^{m} / c_{\phi}^{2}$ respectively. $\left(C_{32}^{\alpha \beta}\right)^{(0)}$ can be obtained from $\left(C_{31}^{\alpha \beta}\right)^{(0)}$ by using the $\lambda_{1,2}-\psi$ interchange symmetry (eq. (2.36)) i.e. $\lambda_{1} \leftrightarrow \lambda_{2}, c_{\psi}^{2} \leftrightarrow s_{\psi}^{2}$ and $s_{\psi} c_{\psi} \rightarrow-s_{\psi} c_{\psi}$, which also changes the sign on the $J^{m}$ 's.

\begin{tabular}{|c|c|c|c|}
\hline$\nu_{\alpha} \rightarrow \nu_{\beta}$ & $F_{1}^{\alpha \beta}$ & $G_{1}^{\alpha \beta}$ & $K_{1}^{\alpha \beta}$ \\
\hline$\nu_{e} \rightarrow \nu_{e}$ & $-2 c_{\phi}^{3} s_{\phi} s_{\psi}^{3} c_{\psi}$ & $2 s_{\phi} c_{\phi} s_{\psi} c_{\psi} c_{2 \phi}$ & 0 \\
\hline$\nu_{\mu} \rightarrow \nu_{e}$ & $\begin{array}{c}c_{\phi} s_{\psi}^{2}\left[s_{\phi} s_{\psi} c_{\psi}\left(c_{23}^{2}+c_{2 \phi} s_{23}^{2}\right)\right. \\
\left.-s_{23} c_{23}\left(s_{\phi}^{2} s_{\psi}^{2}+c_{2 \phi} c_{\psi}^{2}\right) \cos \delta\right]\end{array}$ & $-2 s_{\phi} c_{\phi} s_{\psi}\left(s_{23}^{2} c_{2 \phi} c_{\psi}-s_{23} c_{23} s_{\phi} s_{\psi} \cos \delta\right)$ & $-s_{23} c_{23} c_{\phi} s_{\psi}^{2}\left(c_{\phi}^{2} c_{\psi}^{2}-s_{\phi}^{2}\right) \sin \delta$ \\
\hline$\nu_{\mu} \rightarrow \nu_{\mu}$ & $\begin{array}{c}2 c_{\phi} s_{\psi}\left(s_{23}^{2} s_{\phi} c_{\psi}+s_{23} c_{23} s_{\psi} \cos \delta\right) \times \\
\left(c_{23}^{2} c_{\psi}^{2}-2 s_{23} c_{23} s_{\phi} s_{\psi} c_{\psi} \cos \delta+s_{23}^{2} s_{\phi}^{2} s_{\psi}^{2}\right)\end{array}$ & $\begin{array}{c}-2 c_{\phi} s_{\psi}\left(s_{23}^{2} s_{\phi} c_{\psi}+s_{23} c_{23} s_{\psi} \cos \delta\right) \\
\times\left(1-2 c_{\phi}^{2} s_{23}^{2}\right)\end{array}$ & 0 \\
\hline
\end{tabular}

Table 2. The functions $F_{1}^{\alpha \beta}, G_{1}^{\alpha \beta}$ and $K_{1}^{\alpha \beta}$, from eq. (4.8), are used to calculate the first order coefficients $\left(C_{i j}^{\alpha \beta}\right)^{(1)}$ and $\left(D^{\alpha \beta}\right)^{(1)}$ through eq. (4.7). $F_{2}^{\alpha \beta}, G_{2}^{\alpha \beta}$ and $K_{2}^{\alpha \beta}$ can be obtained using the $\lambda_{1,2}-\psi$ interchange symmetry (eq. (2.36)) i.e. $\lambda_{1} \leftrightarrow \lambda_{2}, c_{\psi}^{2} \leftrightarrow s_{\psi}^{2}$ and $s_{\psi} c_{\psi} \rightarrow-s_{\psi} c_{\psi}$. The angles in matter, $\phi, \psi$, are given in sections 2.3 and 2.4. 


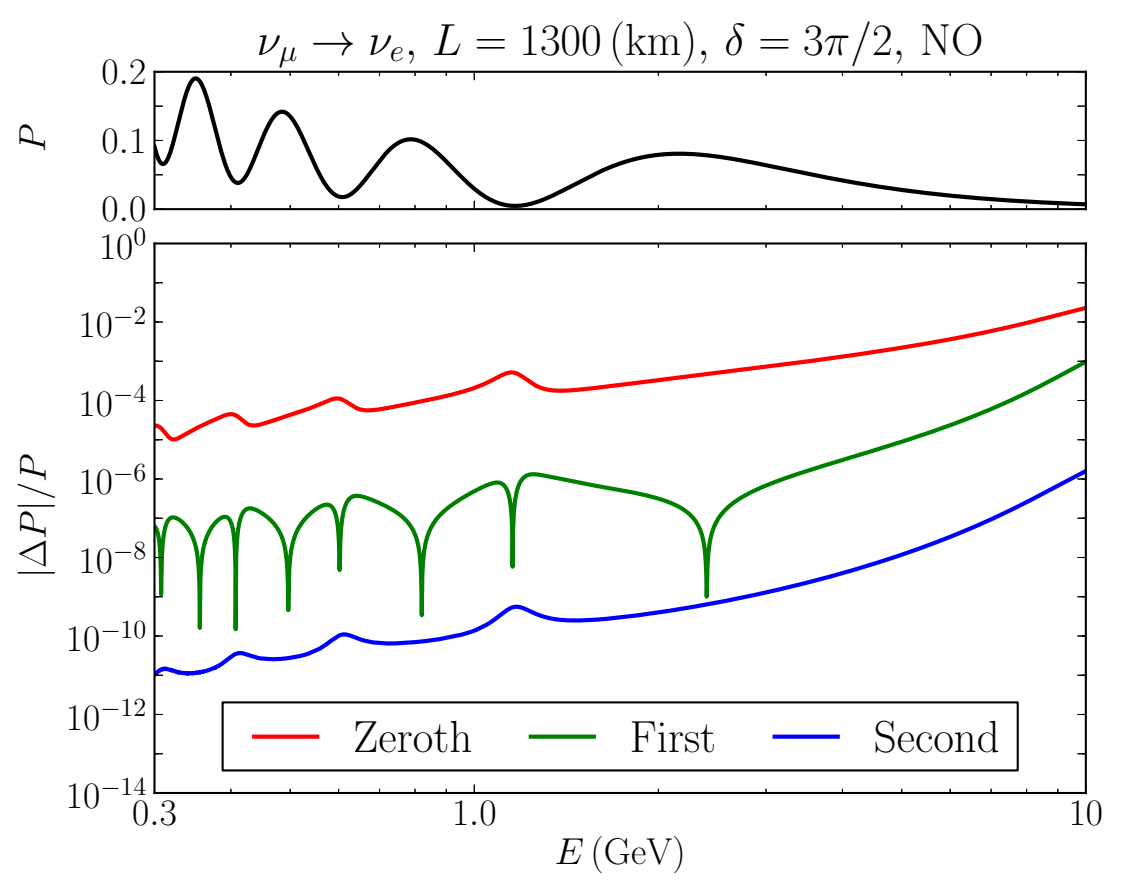

Figure 3. The $\nu_{\mu} \rightarrow \nu_{e}$ oscillation probability is plotted in the upper part of the figure for DUNE parameters; a $1300 \mathrm{~km}$ baseline and $Y_{e} \rho=1.4 \mathrm{~g} \cdot \mathrm{cm}^{-3}$. The fractional uncertainties at zeroth and first order are plotted using the analytic formulas in tables 1 and 2 respectively. The probability to second order is calculated by using $\lambda$ 's and $W$ through second order, see eqs. (3.3) and (3.9).

\subsection{Precision of the perturbation expansion}

The oscillation probabilities that were perturbatively calculated in this section are only useful if they are more precise than the experimental uncertainties. In figure 3, we have plotted the fractional uncertainties ${ }^{8}$ at each order of our perturbative expansion for the $\nu_{\mu} \rightarrow \nu_{e}$ channel at the DUNE [17], baseline of $1300 \mathrm{~km}$. The precision at the first oscillation maximum and minimum for DUNE are shown in table 3. We note that the precision improves at lower energies, such as for $\mathrm{NO} \nu \mathrm{A}[18]$ and $\mathrm{T} 2 \mathrm{~K} / \mathrm{T} 2 \mathrm{HK}[19,20]$. The results are comparable for different values of $\delta$, for the inverted ordering, for other channels, and for antineutrino mode. Therefore, even at zeroth order, the precision exceeds the precision of the expected experimental results.

The oscillation probabilities of [11] started to become less accurate when

$$
|a|<\frac{1}{3} \Delta m_{e e}^{2} \quad \text { and } \quad \frac{L}{E}>1000 \mathrm{~km} / \mathrm{GeV},
$$

as note therein. This restriction is removed in this paper as the eigenvalues no longer cross at the solar resonance. This improves the accuracy of the oscillation probabilities for $\mathrm{T} 2 \mathrm{~K} / \mathrm{T} 2 \mathrm{HK}, \mathrm{NO} \nu \mathrm{A}$ and DUNE. Also, for example, one could use the $\bar{\nu}_{e} \rightarrow \bar{\nu}_{e}$ disappearance probabilities of this paper to quantify the size of the matter effect for the medium baseline

\footnotetext{
${ }^{8}$ The exact oscillation probability were calculated using $[3,4]$.
} 


\begin{tabular}{|c|c|c|c|}
\hline \multicolumn{2}{|c|}{ DUNE: NO, $\delta=3 \pi / 2$} & First min & First max \\
\hline \multicolumn{2}{|c|}{$P\left(\nu_{\mu} \rightarrow \nu_{e}\right)$} & 0.0047 & 0.081 \\
\hline \multicolumn{2}{|c|}{$E(\mathrm{GeV})$} & 1.2 & 2.2 \\
\hline \multirow{2}{*}{$\frac{|\Delta P|}{P}$} & Zeroth & $5 \times 10^{-4}$ & $4 \times 10^{-4}$ \\
\cline { 2 - 4 } & First & $3 \times 10^{-7}$ & $2 \times 10^{-7}$ \\
\cline { 2 - 4 } & Second & $6 \times 10^{-10}$ & $5 \times 10^{-10}$ \\
\hline
\end{tabular}

Table 3. The transition probabilities, energies, and fractional uncertainties at zeroth, first, and second order. Values are calculated at DUNE for $\nu_{\mu} \rightarrow \nu_{e}$ with the NO and $\delta=3 \pi / 2$. At higher maxima and minima the fractional uncertainties are even smaller.

experiments JUNO, [21], and RENO-50, [22, 23], a setup where the oscillation probabilities of [11] miss significant physics, since the $L / E$ varies from 6 to $25 \mathrm{~km} / \mathrm{MeV}$.

\section{Conclusions}

In this paper we have further developed and expanded upon the recent perturbative framework for neutrino oscillations in uniform matter, introduced in [11]. The new oscillation probabilities are of the same simple, compact functional form with slightly more complicated coefficients, yet, the range of applicability now includes the whole $L / E$ versus matter potential, $a$, plane, i.e. the restriction that $L / E$ be small, $\left(L / E \ll 1 / \Delta m_{21}^{2}\right)$ around the vacuum values of the matter potential has been completely removed. In fact, with these new improvements, the oscillation probabilities in vacuum are exact at zeroth order in our perturbative expansion. This occurs because the expansion parameter $s_{12} c_{12} \Delta m_{21}^{2} / \Delta m_{e e}^{2}=0.014$ is further multiplied by $s_{\left(\phi-\theta_{13}\right)}$, where $\phi$ is the mixing angle $\theta_{13}$ in matter. In vacuum, $\phi=\theta_{13}$ and therefore all corrections to zeroth order vanish.

To achieve this extended range of applicability, an additional rotation of the Hamiltonian is performed over that in [11]. The third angle $\psi$ is the mixing angle $\theta_{12}$ in matter. In the resulting Hamiltonian, the diagonal elements are the eigenvalues of the zeroth order Hamiltonian and do not cross for any values of the matter potential, especially near the solar resonance (this occurred in [11]). The non-diagonal elements of the new Hamiltonian are the perturbing Hamiltonian for our perturbative expansion and their size is controlled by the small parameter $s_{\left(\phi-\theta_{13}\right)} s_{12} c_{12} \Delta m_{21}^{2} / \Delta m_{e e}^{2}$, mentioned in the previous paragraph. The new perturbative expansion is now well defined for all values of the matter potential and gives very accurate oscillation probabilities. We have performed many cross checks on the perturbative expansion, e.g. we have checked the $C P$ violating term recovers, order by order, the known form. We have calculated the oscillation probabilities for zeroth, first, and second order in our expansion parameter. For most practical applications related to experiments, the zeroth order oscillation probabilities are sufficiently accurate with a typical fractional uncertainty of better than $10^{-3}$. Including the first and second order corrections the accuracy improves that to better than $10^{-6}$ and $10^{-9}$, respectively. 


\section{Acknowledgments}

P.B.D. acknowledges support from the Fermilab Graduate Student Research Program in Theoretical Physics operated by Fermi Research Alliance, LLC. This work is also supported in part by DOE grant DE-SC0011981.

H.M. thanks Instituto de Física, Universidade de São Paulo for the great opportunity of stay under support by Fundação de Amparo à Pesquisa do Estado de São Paulo (FAPESP) with grant number 2015/05208-4. He thanks Fermilab Theory Group for warm hospitality in his visits.

S.P. acknowledges partial support from the European Union FP7 ITN INVISIBLES (Marie Curie Actions, PITN-GA-2011-289442). This project has received funding from the European Union's Horizon 2020 research and innovation programme under the Marie Skłodowska-Curie grant agreement No 690575-InvisiblesPlus RISE. This project has received funding from the European Union's Horizon 2020 research and innovation programme under the Marie Skłodowska-Curie grant agreement No 674896-Elusives ITN.

Fermilab is operated by the Fermi Research Alliance, LLC under contract no. DEAC02-07CH11359 with the U.S. Department of Energy.

\section{A Technical details}

\section{A.1 Generalized approach to diagonalization}

We describe the diagonalization of a particular $2 \times 2$ submatrix and the angle and eigenvalues. This is the approach used twice in subsections 2.3 and 2.4 to diagonalize the 1-3 and then the 1-2 submatrices.

Given a general symmetric $2 \times 2$ matrix we wish to diagonalize with angle $\phi$, we write

$$
\left(\begin{array}{cc}
\lambda_{\sigma} & \\
& \lambda_{\rho}
\end{array}\right)=U(\phi)^{\dagger}\left(\begin{array}{cc}
\lambda_{a} & \lambda_{x} \\
\lambda_{x} & \lambda_{c}
\end{array}\right) U(\phi),
$$

where

$$
U(\phi) \equiv\left(\begin{array}{cc}
c_{\phi} & s_{\phi} \\
-s_{\phi} & c_{\phi}
\end{array}\right) .
$$

Since trace and determinant are unchanged by the $U$ sandwich,

$$
\lambda_{\sigma}+\lambda_{\rho}=\lambda_{a}+\lambda_{c} \quad \text { and } \quad \lambda_{\rho} \lambda_{\sigma}=\lambda_{a} \lambda_{c}-\lambda_{x}^{2}
$$

By squaring the trace equation and subtracting 4 times the determinant equation we have

$$
\left(\lambda_{\rho}-\lambda_{\sigma}\right)^{2}=\left(\lambda_{a}-\lambda_{c}\right)^{2}+4 \lambda_{x}^{2}
$$

thus

$$
\lambda_{\rho, \sigma}=\frac{1}{2}\left[\left(\lambda_{a}+\lambda_{c}\right) \pm \sqrt{\left(\lambda_{a}-\lambda_{c}\right)^{2}+4 \lambda_{x}^{2}}\right] .
$$


Next, we rewrite eq. (A.1) by left (right) multiplying by $U(\phi)\left(U^{\dagger}(\phi)\right)$, then

$$
U(\phi)\left(\begin{array}{cc}
\lambda_{\sigma} & \\
& \lambda_{\rho}
\end{array}\right) U(\phi)^{\dagger}=\left(\begin{array}{cc}
c_{\phi}^{2} \lambda_{\sigma}+s_{\phi}^{2} \lambda_{\rho} & s_{\phi} c_{\phi}\left(\lambda_{\rho}-\lambda_{\sigma}\right) \\
s_{\phi} c_{\phi}\left(\lambda_{\rho}-\lambda_{\sigma}\right) & s_{\phi}^{2} \lambda_{\sigma}+c_{\phi}^{2} \lambda_{\rho}
\end{array}\right)=\left(\begin{array}{cc}
\lambda_{a} & \lambda_{x} \\
\lambda_{x} & \lambda_{c}
\end{array}\right) .
$$

This gives us three equations,

$$
\begin{aligned}
\lambda_{a} & =c_{\phi}^{2} \lambda_{\sigma}+s_{\phi}^{2} \lambda_{\rho}, \\
\lambda_{c} & =s_{\phi}^{2} \lambda_{\sigma}+c_{\phi}^{2} \lambda_{\rho}, \\
\lambda_{x} & =\left(\lambda_{\rho}-\lambda_{\sigma}\right) s_{\phi} c_{\phi} .
\end{aligned}
$$

The last equation is the standard equation for $s_{2 \phi}$. Subtracting (adding) the first two gives the standard equation for $c_{2 \phi}$ (the trace). Thus the rotation angle is defined by the following

$$
\lambda_{x}=\left(\lambda_{\rho}-\lambda_{\sigma}\right) s_{\phi} c_{\phi} \quad \text { and } \quad\left(\lambda_{c}-\lambda_{a}\right)=\left(\lambda_{\rho}-\lambda_{\sigma}\right)\left(c_{\phi}^{2}-s_{\phi}^{2}\right) .
$$

In addition, using only $c_{\phi}^{2}+s_{\phi}^{2}=1$ we can write down the following useful identities

$$
\begin{aligned}
& c_{\phi}^{2}=\frac{\lambda_{\rho}-\lambda_{a}}{\lambda_{\rho}-\lambda_{\sigma}}=\frac{\lambda_{c}-\lambda_{\sigma}}{\lambda_{\rho}-\lambda_{\sigma}}, \\
& s_{\phi}^{2}=\frac{\lambda_{\rho}-\lambda_{c}}{\lambda_{\rho}-\lambda_{\sigma}}=\frac{\lambda_{a}-\lambda_{\sigma}}{\lambda_{\rho}-\lambda_{\sigma}},
\end{aligned}
$$

which are used extensively throughout this paper. This set of operations will be used both for $\phi$ and $\psi$ rotations.

\section{A.2 Useful identities}

From the trace and determinant identities, see eq. (A.3),

$$
\begin{aligned}
\lambda_{-}+\lambda_{+} & =\lambda_{a}+\lambda_{c}, \\
\lambda_{1}+\lambda_{2} & =\lambda_{-}+\lambda_{0}, \\
\lambda_{+} \lambda_{-} & =\lambda_{a} \lambda_{c}-\left[\Delta m_{e e}^{2} c_{13} s_{13}\right]^{2}, \\
\lambda_{1} \lambda_{2} & =\lambda_{0} \lambda_{-}-\left[\epsilon \Delta m_{e e}^{2} c_{12} s_{12} c_{\left(\phi-\theta_{13}\right)}\right]^{2},
\end{aligned}
$$

where we recall that the $\lambda_{a, b, c}$ in the tilde basis are defined in eq. (2.12). Another useful relation is

$$
c_{\left(\phi-\theta_{13}\right)} s_{\left(\phi-\theta_{13}\right)}=s_{13} c_{13} \frac{a}{\Delta \lambda_{+-}},
$$

then for $a \ll \Delta m_{e e}^{2}$,

$$
s_{\left(\phi-\theta_{13}\right)} \approx s_{13} c_{13} \frac{a}{\Delta m_{e e}^{2}}
$$

\section{A.3 Limits}

We list the values of the angles and the eigenvalues in vacuum and for $a \rightarrow \pm \infty$ in table 4 . 


\begin{tabular}{|c|c|c|c|}
\hline$a$ & 0 & $-\infty$ & $+\infty$ \\
\hline$\phi$ & $\theta_{13}$ & $0(\pi / 2)$ & $\pi / 2(0)$ \\
$\psi$ & $\theta_{12}$ & 0 & $\pi / 2$ \\
\hline$\lambda_{-}$ & $s_{12}^{2} \Delta m_{21}^{2}$ & $\lambda_{a}\left(\lambda_{c}\right)$ & $\lambda_{c}\left(\lambda_{a}\right)$ \\
$\lambda_{0}$ & $c_{12}^{2} \Delta m_{21}^{2}$ & $\lambda_{b}$ & $\lambda_{b}$ \\
$\lambda_{+}$ & $\Delta m_{31}^{2}$ & $\lambda_{c}\left(\lambda_{a}\right)$ & $\lambda_{a}\left(\lambda_{c}\right)$ \\
\hline$\lambda_{1}$ & 0 & $\lambda_{a}\left(\lambda_{c}\right)$ & $\lambda_{b}$ \\
$\lambda_{2}$ & $\Delta m_{21}^{2}$ & $\lambda_{b}$ & $\lambda_{c}\left(\lambda_{a}\right)$ \\
$\lambda_{3}$ & $\Delta m_{31}^{2}$ & $\lambda_{c}\left(\lambda_{a}\right)$ & $\lambda_{a}\left(\lambda_{c}\right)$ \\
\hline
\end{tabular}

Table 4. The NO (IO) limits of the angles and the eigenvalues in vacuum and for $a \rightarrow \pm \infty$, where $\lambda_{a}=a+\left(s_{13}^{2}+\epsilon s_{12}^{2}\right) \Delta m_{e e}^{2}, \lambda_{b}=\epsilon c_{12}^{2} \Delta m_{e e}^{2}$, and $\lambda_{c}=\left(c_{13}^{2}+\epsilon s_{12}^{2}\right) \Delta m_{e e}^{2}$, from eq. (2.12).

\section{A.4 Characteristic equation}

The characteristic equation for neutrino oscillation in matter is

$$
\begin{aligned}
\lambda^{3}- & \left(\Delta m_{21}^{2}+\Delta m_{31}^{2}+a\right) \lambda^{2}+\left\{\Delta m_{21}^{2} \Delta m_{31}^{2}+a\left[\left(c_{12}^{2}+s_{12}^{2} s_{13}^{2}\right) \Delta m_{21}^{2}+c_{13}^{2} \Delta m_{31}^{2}\right]\right\} \lambda \\
-\left(a c_{12}^{2} c_{13}^{2} \Delta m_{21}^{2} \Delta m_{31}^{2}\right) & =0 .
\end{aligned}
$$

The coefficient of the $\lambda^{2}$ term is the sum of the eigenvalues, the coefficient of the $\lambda$ term is the sum of pairs of the eigenvalues, and the coefficient of the $\lambda^{0}$ term is the triple product of eigenvalues.

We now verify that our matter mass eigenvalues satisfy these expressions to second order. First, the $\lambda_{-, 0,+}$ eigenvalues satisfy the first requirement exactly as was discussed in [11]. Since $\sum_{i=1,2,3} \lambda_{i}=\sum_{i=-, 0,+} \lambda_{i}$, so the $\lambda_{1,2,3}$ eigenvalues also satisfy the first requirement. Also, from eq. (3.3), $\sum_{i=1,2,3} \lambda_{i}^{(2)}=0$, so the $\lambda_{1,2,3}$ eigenvalues also satisfy the first requirement exactly through second order. We have also verified that each of the other two conditions are satisfied to second order.

\section{A.5 Unitarity of the $W$ matrix}

We verify that the $V$ matrix satisfies the unitarity requirements, $V V^{\dagger}=\mathbb{1} . U_{\text {MNS }}^{m}$ is unitary by definition. Then we just need that the $W$ matrix is unitary. The zeroth order requirement is $W_{0} W_{0}^{\dagger}=\mathbb{1}$ which is immediately satisfied since $W_{0}=\mathbb{1}$. At first order the requirement is $W_{1}+W_{1}^{\dagger}=0$. This is equivalent, to the requirement that $W_{1}$ is antiHermitian, or that $\check{H}_{1}$ is Hermitian, which they are, respectively, see eq. (3.8).

To second order, the unitarity requirement becomes, $W_{2}+W_{2}^{\dagger}=-W_{1}^{2}$. That is, that the Hermitian part of $W_{2}$ must be $-W_{1}^{2} / 2$, which it is. An additional anti-Hermitian part is unconstrained and is calculated through perturbation theory. 


\section{A.6 $V$-matrix, $S$-matrix comparison}

In the S-matrix method, the oscillation probabilities are given by, see for example [11],

$$
\begin{aligned}
S_{S}(L)= & U_{\mathrm{MNS}}^{m} e^{-i H_{0} L} \Omega(L)\left(U_{\mathrm{MNS}}^{m}\right)^{\dagger} \\
\Omega(L)= & 1+(-i) \int_{0}^{L} d x e^{i H_{0} x} H_{1} e^{-i H_{0} x} \\
& +(-i)^{2} \int_{0}^{L} d x e^{i H_{0} x} H_{1} e^{-i H_{0} x} \int_{0}^{x} d x^{\prime} e^{i H_{0} x^{\prime}} H_{1} e^{-i H_{0} x^{\prime}}+\cdots,
\end{aligned}
$$

where $H_{0}$ and $H_{1}$ are given by eqs. (2.23) and (2.24). (We drop the "check" in this appendix.)

In the V-matrix method, used in this paper, the oscillation probabilities are given by,

$$
\begin{aligned}
S_{V}(L) & =U_{\mathrm{MNS}}^{m} W e^{-i \Lambda L / 2 E} W^{\dagger}\left(U_{\mathrm{MNS}}^{m}\right)^{\dagger} \\
(\Lambda)_{i j} & =\delta_{i j}\left(\lambda_{i}+\lambda_{i}^{(1)}+\lambda_{i}^{(2)}+\cdots\right) \\
W & =1+W_{1}+W_{2}+\cdots
\end{aligned}
$$

where the $\lambda_{i} / 2 E$ are the eigenvalues of $H_{0} . \lambda_{i}^{(n)}$ and $W_{n}$ are given by n-th order perturbation theory.

Specializing to the case when the perturbing Hamiltonian has no diagonal elements,

$$
\left(H_{1}\right)_{i j}=\left(1-\delta_{i j}\right) h_{i j} / 2 E
$$

which is relevant for the perturbation discussed in this paper, $W$ can be calculated from eq. (3.8) for first order and eq. (3.9) for second order.

Then it is trivial to show that to first order,

$$
\begin{aligned}
{\left[\left(U_{\mathrm{MNS}}^{m}\right)^{\dagger} S_{S}(L) U_{\mathrm{MNS}}^{m}\right]_{i j} } & =\left[\left(U_{\mathrm{MNS}}^{m}\right)^{\dagger} S_{V}(L) U_{\mathrm{MNS}}^{m}\right]_{i j} \\
& =\delta_{i j} e^{-i \lambda_{i} L / 2 E}+\left(1-\delta_{i j}\right) \frac{h_{i j}}{\Delta \lambda_{i j}}\left(e^{-i \lambda_{i} L / 2 E}-e^{-i \lambda_{j} L / 2 E}\right) .
\end{aligned}
$$

We have also checked that they are equal at second order. As this is just a consistency check of perturbation theory, we postulate that it is true to all orders, without presenting an all orders proof.

\section{A.7 $\quad C P$ violating term}

It is useful to rewrite the numerator of eq. (4.9) as $\epsilon\left(\Delta m_{e e}^{2}\right)^{3}\left(1-\epsilon \cos 2 \theta_{12}-\epsilon^{2} c_{12}^{2} s_{12}^{2}\right)$. We evaluate $D^{e \mu}$ through first order, keeping terms that are explicitly second order in $\epsilon$, noting that dividing by $\Delta \lambda_{21}$ introduces an additional factor of $\epsilon$ in vacuum.

$$
\left(D^{e \mu}\right)^{(0)}+\left(D^{e \mu}\right)^{(1)}=s_{\delta} J_{r} \frac{\epsilon\left(\Delta m_{e e}^{2}\right)^{3}\left(1-\epsilon \cos 2 \theta_{12}\right)}{\Delta \lambda_{21} \Delta \lambda_{31} \Delta \lambda_{32}},
$$

where $J_{r}$ is the reduced Jarlskog factor, see ref. [24],

$$
J_{r} \equiv c_{12} s_{12} c_{13}^{2} s_{13} c_{23} s_{23} .
$$


The dropped higher order contribution to the numerator is

$$
\begin{aligned}
& -\epsilon^{2} c_{12}^{2} s_{12}^{2} \frac{\Delta \lambda_{+-}+\left(\Delta m_{e e}^{2}-a\right)}{4\left(\Delta \lambda_{+-}\right)^{3}} \\
& \quad \times\left[\left(\Delta m_{e e}^{2}\right)^{2}+3 a^{2}-4 c_{2 \theta_{13}} a \Delta m_{e e}^{2}+\left(\Delta m_{e e}^{2}+a\right) \Delta \lambda_{+-}\right],
\end{aligned}
$$

which is $-\epsilon^{2} c_{12}^{2} s_{12}^{2}$ in vacuum as desired since $\Delta \lambda_{+-}$is $\Delta m_{e e}^{2}$ in vacuum.

Open Access. This article is distributed under the terms of the Creative Commons Attribution License (CC-BY 4.0), which permits any use, distribution and reproduction in any medium, provided the original author(s) and source are credited.

\section{References}

[1] L. Wolfenstein, Neutrino Oscillations in Matter, Phys. Rev. D 17 (1978) 2369 [InSPIRE].

[2] V.D. Barger, K. Whisnant, S. Pakvasa and R.J.N. Phillips, Matter Effects on Three-Neutrino Oscillations, Phys. Rev. D 22 (1980) 2718 [INSPIRE].

[3] H.W. Zaglauer and K.H. Schwarzer, The Mixing Angles in Matter for Three Generations of Neutrinos and the MSW Mechanism, Z. Phys. C 40 (1988) 273 [INSPIRE].

[4] K. Kimura, A. Takamura and H. Yokomakura, Exact formulas and simple CP dependence of neutrino oscillation probabilities in matter with constant density, Phys. Rev. D 66 (2002) 073005 [hep-ph/0205295] [INSPIRE].

[5] M. Blennow and A.Yu. Smirnov, Neutrino propagation in matter, Adv. High Energy Phys. 2013 (2013) 972485 [arXiv:1306.2903] [INSPIRE].

[6] E.K. Akhmedov and V. Niro, An accurate analytic description of neutrino oscillations in matter, JHEP 12 (2008) 106 [arXiv:0810.2679] [INSPIRE].

[7] A. Cervera et al., Golden measurements at a neutrino factory, Nucl. Phys. B 579 (2000) 17 [Erratum ibid. B 593 (2001) 731] [hep-ph/0002108] [INSPIRE].

[8] J. Arafune and J. Sato, $C P$ and T violation test in neutrino oscillation, Phys. Rev. D 55 (1997) 1653 [hep-ph/9607437] [INSPIRE].

[9] M. Freund, Analytic approximations for three neutrino oscillation parameters and probabilities in matter, Phys. Rev. D 64 (2001) 053003 [hep-ph/0103300] [INSPIRE].

[10] E.K. Akhmedov, R. Johansson, M. Lindner, T. Ohlsson and T. Schwetz, Series expansions for three flavor neutrino oscillation probabilities in matter, JHEP 04 (2004) 078 [hep-ph/0402175] [INSPIRE].

[11] H. Minakata and S.J. Parke, Simple and Compact Expressions for Neutrino Oscillation Probabilities in Matter, JHEP 01 (2016) 180 [arXiv:1505.01826] [INSPIRE].

[12] H. Nunokawa, S.J. Parke and R. Zukanovich Funchal, Another possible way to determine the neutrino mass hierarchy, Phys. Rev. D 72 (2005) 013009 [hep-ph/0503283] [INSPIRE].

[13] S. Parke, What is $\Delta m_{e e}^{2}$ ?, Phys. Rev. D 93 (2016) 053008 [arXiv: 1601.07464] [InSPIRE].

[14] H. Minakata and $\mathrm{H}$. Nunokawa, CP violation versus matter effect in long baseline neutrino oscillation experiments, Phys. Rev. D 57 (1998) 4403 [hep-ph/9705208] [INSPIRE]. 
[15] V.A. Naumov, Three neutrino oscillations in matter, CP-violation and topological phases, Int. J. Mod. Phys. D 1 (1992) 379 [inSPIRE].

[16] P.F. Harrison and W.G. Scott, $C P$ and T violation in neutrino oscillations and invariance of Jarlskog's determinant to matter effects, Phys. Lett. B 476 (2000) 349 [hep-ph/9912435] [INSPIRE].

[17] DUNE collaboration, R. Acciarri et al., Long-Baseline Neutrino Facility (LBNF) and Deep Underground Neutrino Experiment (DUNE) Conceptual Design Report Volume 2: The Physics Program for DUNE at LBNF, arXiv:1512.06148 [INSPIRE].

[18] NOvA collaboration, R.B. Patterson, The NOvA Experiment: Status and Outlook, arXiv: 1209.0716 [INSPIRE].

[19] T2K collaboration, K. Abe et al., The T2K Experiment, Nucl. Instrum. Meth. A 659 (2011) 106 [arXiv: 1106.1238] [INSPIRE].

[20] Hyper-Kamiokande Proto-collaboration, K. Abe et al., Physics potential of a long-baseline neutrino oscillation experiment using a J-PARC neutrino beam and Hyper-Kamiokande, PTEP 2015 (2015) 053C02 [arXiv:1502.05199] [INSPIRE].

[21] JUNO collaboration, F. An et al., Neutrino Physics with JUNO, J. Phys. G 43 (2016) 030401 [arXiv: 1507.05613] [INSPIRE].

[22] S.-B. Kim, Next generation reactor neutrino physics, in proceedings of International Workshop on RENO-50 towards Neutrino Mass Hierarchy, Seoul, South Korea, 13-14 June 2013, http://home.kias.re.kr/MKG/h/reno50/.

[23] S.-H. Seo, Introduction to RENO-50, in proceedings of International Workshop on RENO-50 towards Neutrino Mass Hierarchy, Seoul, South Korea, 13-14 June 2013, http://home.kias.re.kr/MKG/h/reno50/.

[24] C. Jarlskog, Commutator of the Quark Mass Matrices in the Standard Electroweak Model and a Measure of Maximal CP-violation, Phys. Rev. Lett. 55 (1985) 1039 [INSPIRE]. 